Fonoaudiologia Baseada

em Evidências

Evidence-based Speech-Language

Pathology and Audiology

Maria Inês Vieira Couto ${ }^{1}$ Ana Claudia Martinho Carvalho

Descritores

Reabilitação

Implante coclear

Pais

Relações pais-filho

Criança

Keywords

Rehabilitation

Cochlear implantation

Parents

Parent-child relations

Child

Endereço para correspondência:

Maria Inês Vieira Couto

R. Cipotânea, 51, Cidade Universitária, São Paulo (SP), Brasil, CEP: 05360-160.

E-mail: micouto@usp.br

Recebido em: 17/1/2012

Aceito em: 17/8/2012

\section{Fatores que influenciam na participação dos pais de crianças usuárias de implante coclear na (re)habilitação \\ oral: revisão sistemática}

\author{
Factors that influence the participation of parents in the oral \\ rehabilitation process of children with cochlear implants: a \\ systematic review
}

\section{RESUMO}

Objetivo: Identificar e analisar os fatores que interferem na participação dos pais de crianças usuárias de implante coclear (IC) no processo (re)habilitação aurioral. Estratégia de pesquisa: Formulação da pergunta da pesquisa, levantamento e seleção dos estudos em bases de dados, com os descritores cochlear implant (implante coclear) e parents (pais). Critérios de seleção: Artigos originais e completos publicados na língua portuguesa brasileira ou inglesa, pais de crianças usuárias de IC como participantes diretos da pesquisa. Análise dos dados: Leitura dos artigos na íntegra e extração de dados para a caracterização dos centros, da metodologia e conteúdo das pesquisas. Resultados: Treze artigos foram selecionados, dos tipos transversal e caso-controle (técnica de entrevista). Foram identificados os fatores sobre a tomada de decisão em realizar ou não a cirurgia (conhecimento prévio sobre IC, expectativas dos resultados, qualidade e quantidade de informações, encaminhamentos realizados, aspectos biomédicos e éticos, inserção na reabilitação, contato com outros pais, apoio social e custos); os fatores preditivos relacionados aos resultados da reabilitação (tempo de uso diário do IC, modalidade aurioral, escola regular, comprometimentos associados, aspectos sócio-demográficos e efetividade do programa); os fatores relacionados aos benefícios e limitações do IC (modalidade comunicativa, evolução das habilidades auditivas e linguísticas, o aprendizado de uma segunda língua oral), bem como o grau de satisfação dos pais. Conclusão: O engajamento dos pais de crianças usuárias de IC na (re)habilitação aurioral depende de fatores relacionados a diferentes domínios, os quais os fonoaudiólogos devem compreender e considerar na elaboração do programa de intervenção.

\begin{abstract}
Purpose: To identify and analyze factors that influence the participation of parents in the rehabilitation process of children with cochlear implants (CI). Research strategy: Question formulation and articles selection in three databases using the following keywords: cochlear implant (implante coclear) and parents (pais). Selection criteria: Complete original articles published in Brazilian Portuguese or English, with direct participation of parents of children with CI. Data analysis: Articles were fully read. Data regarding characterization of the centers, research methodology and content were analyzed. Results: Thirteen articles were selected based on the established criteria. The types of studies were cross-sectional and case-control (interview technique). The following influential factors were identified: pre-CI surgery factors (knowledge about CI, quality and quantity of information, specialist's advices, ethical and biomedical aspects, rehabilitation engagement, contact with experienced families, social service support and overall costs); rehabilitation aspects (CI use, oral communication modality, regular school, other disabilities, social and demographic aspects and rehabilitation program's effectiveness); other important influential processes (communication modality, auditory and language development, second oral language learning, as well as parent's behavior and satisfaction). Conclusion: The engagement of parents in the rehabilitation process of children with $\mathrm{CI}$ depends on several distinct influential factors which audiologists should understand and consider when elaborating a rehabilitation program.
\end{abstract}

Trabalho realizado no Departamento de Fisioterapia, Fonoaudiologia e Terapia Ocupacional, Faculdade de Medicina, Universidade de São Paulo - USP - São Paulo (SP), Brasil.

(1) Departamento de Fisioterapia, Fonoaudiologia e Terapia Ocupacional, Faculdade de Medicina, Universidade de São Paulo - USP - São Paulo (SP), Brasil.

Conflito de interesses: Não 


\section{INTRODUÇÃO}

A (re)habilitação aurioral pediátrica tem como propósito desenvolver a língua falada e aprimorar a fluência conversacional da criança com perda auditiva por meio da audição, direcionando-a para a independência e integração na comunidade ouvinte ${ }^{(1-3)}$. No caso de criança com perda auditiva neurossensorial bilateral de graus severo e/ou profundo, após avaliação completa de uma equipe multidisciplinar, o implante coclear (IC) pode ser o dispositivo eletrônico selecionado.

Este efetivo recurso clínico caracteriza-se por ser uma prótese de sofisticada tecnologia, que é inserida cirurgicamente na cóclea, com o objetivo de substituir parcialmente as funções do órgão de Corti. Fornece impulsos elétricos para a estimulação direta das fibras neurais remanescentes na cóclea, possibilitando acesso aos sons da fala ${ }^{(4)}$.

Evidências na literatura apontam que a variabilidade dos resultados do IC em crianças está associada a diversos fatores, tais como: etiologia, idade da ativação do IC, quantidade de resíduo auditivo antes da cirurgia, tempo de privação sensorial, número de eletrodos inseridos na cóclea, tempo diário do uso do dispositivo e o envolvimento dos pais no processo de (re) habilitação auditiva ${ }^{(5-7)}$.

Um dos mais importantes fatores que interfere no desenvolvimento da criança é a participação dos pais, uma vez que está diretamente relacionada aos resultados de avaliação das habilidades comunicativas e linguísticas da criança com perda auditiva. O envolvimento dos pais pode ser caracterizado pelos seguintes comportamentos: garantir que a criança utilize o IC regularmente; garantir que o IC esteja funcionando apropriadamente; proporcionar estimulação auditiva e de fala de forma consistente e com um bom modelo de linguagem em todos os ambientes; estimular a produção de fala da criança; engajar a criança na conversação; manter contato regular com o professor, participar no desenvolvimento e na implementação do programa de intervenção e frequentar as sessões terapêuticas. Todos estes comportamentos podem ser classificados em uma escala para caracterizar a qualidade de participação dos mesmos ${ }^{(2,5)}$.

Neste cenário, um consenso existe no que se refere a como deve ser a participação dos pais no programa de habilitação e reabilitação. No entanto, os profissionais que atuam com esses pais precisam considerar e disponibilizar condições apropriadas para os pais desempenharem todas as referidas tarefas satisfatoriamente.

Os profissionais da saúde devem ter ciência que logo após o diagnóstico da perda auditiva, os pais, que na grande maioria não tem experiência sobre o assunto, precisam de apoio enquanto se ajustam a esta nova e inesperada situação. Ao mesmo tempo, eles necessitam participar de todo o processo, ou seja, contribuir com a equipe multidisciplinar para que a meta proposta seja alcançada ${ }^{(8)}$. São momentos nos quais os pais passam por muitos conflitos e, ao mesmo tempo, precisam agir.

\section{OBJETIVOS}

Identificar e analisar os fatores que interferem na participação dos pais de crianças usuárias de implante coclear (IC) no processo (re)habilitação aurioral, descrevendo os aspectos metodológicos e os respectivos resultados dos estudos publicados.

\section{ESTRATÉGIA DE PESQUISA}

A primeira etapa consistiu na formulação da pergunta da pesquisa: Quais são os fatores relacionados à participação dos pais que interferem nos resultados (re)habilitação aurioral de crianças usuárias de IC?

Para a seleção dos estudos, foi realizado um levantamento bibliográfico em periódicos nacionais e internacionais pertinentes aos assuntos abordados e disponíveis eletronicamente entre janeiro de 2010 e junho de 2011, nas bases de dados eletrônicos: Medline, LILACS e SciELO. A restrição no período estipulado para seleção dos trabalhos (18 meses) foi realizada considerando-se a ênfase do estudo em detalhar a identificação dos fatores que influenciam a participação dos pais nas diferentes etapas do programa de (re)habilitação.

Os seguintes descritores, em inglês e português, encontrados nos Descritores em Ciência da Saúde (DeCS) foram utilizados: cochlear implant (implante coclear) e parents (pais). A seleção dos estudos publicados foi realizada em três etapas: 1) seleção de materiais com os descritores citados e cruzados (and/e), publicados no período compreendido entre janeiro de 2010 e julho 2011 nas referidas bases de dados e disponíveis eletronicamente; 2) seleção de materiais cujos títulos e resumos estivessem relacionados ao tema proposto; 3 ) análise completa dos artigos.

\section{CRITÉRIOS DE SELEÇÃO}

Os artigos identificados pela estratégia de pesquisa inicial foram avaliados de maneira independente pelas duas autoras, seguindo os critérios de inclusão e exclusão. Foram estabelecidos os seguintes critérios de inclusão dos estudos: ter os pais de crianças com idade inferior a 12 anos e usuárias de IC como participantes diretos; serem os dados coletados do tipo primário (próprios autores coletaram); artigos publicados na língua portuguesa brasileira ou inglesa; e, artigos originais completos.

Levando-se em consideração a classificação dos níveis de evidencia cientifica ${ }^{(9-11)}$, segundo a metodologia dos diferentes tipos de pesquisa, foram excluídos os artigos de estudos de caso (nível de evidência 5) e opiniões de especialistas (de evidências 6), bem como resumos em anais de congressos, dissertações e teses.

\section{ANÁLISE DOS DADOS}

Os artigos selecionados foram lidos na íntegra, sendo extraídos os seguintes dados para a caracterização dos centros de pesquisas e metodologia dos estudos: nome dos autores, ano da publicação; revista publicada, país e instituição onde a pesquisa foi desenvolvida; tamanho da amostra; instrumento utilizado; e, tipo de pesquisa.

Foram coletados os seguintes dados referentes ao conteúdo das pesquisas: objetivo do estudo e as conclusões alcançadas. Posteriormente, foram identificados subtemas abordados nas pesquisas; e, após o levantamento dos resultados e conclusões, 
foi realizada a identificação dos fatores que os profissionais devem considerar quando solicitam a participação dos pais nos diferentes momentos da (re)habilitação aurioral.

As pesquisas selecionadas foram analisadas de acordo com os seguintes aspectos ${ }^{(12,13)}$ :

- Abordagem: quantitativa (utiliza medidas mensuráveis para uma grande população; descrição matemática - teoria da probabilidade), qualitativa (compreensão aprofundada das relações e atividades humanas; valores, crenças, representações, hábitos, atitudes e opiniões de um grupo delimitado - social) ou híbrida (quantitativa e qualitativa).

- Tipo de estudo: transversal (descreve os indivíduos de uma população em relação às suas características) e/ou caso controle (comparação de dois grupos de participantes).

- Tipo de entrevista: estruturada (segue roteiro, geralmente com instrumentos padronizados e respostas de múltipla escolha) e/ou não estruturada (segue roteiro, porém as respostas são do tipo aberta e o entrevistador tem a liberdade para direcioná-la).

- Tipo de instrumento: questionário (perguntas que devem ser respondidas por escrito e sem a presença do entrevistador) e/ou formulário (a coleta de dados consiste em obter informações diretamente do entrevistado por um entrevistador).

\section{RESULTADOS}

\section{Seleção dos estudos analisados}

A pesquisa realizada a partir dos descritores propostos, nas três bases de dados, produziu 220 citações: 213 na base Medline, quatro na base SciELO e três na base LILACS. Após a triagem dos artigos referente ao período estipulado, 111 artigos foram excluídos. Foram lidos os títulos e resumos dos 109 estudos, dos quais 81 não estavam relacionados com o tema proposto; resultaram 28 artigos. Na etapa seguinte, foi realizada a leitura dos artigos completos e foram excluídos 15 artigos, visto que o título e o resumo não esclareciam apropriadamente a metodologia utilizada. No final do levantamento foram selecionados 13 artigos na revisão sistemática (Quadro 1).

\section{Análise dos aspectos metodológicos das pesquisas selecionadas}

\section{Resultados nas bases eletrônicas de dados}

Os periódicos das duas publicações nacionais, na língua portuguesa brasileira, localizadas na base do SciELO, foram Psicologia Teoria e Pesquisa e Arquivos Internacionais de Otorrinolaringologia (Quadro 1).

As outras 11 publicações citadas no Medline, na língua inglesa, foram três artigos na American Annals of The Deaf; dois no Journal of Studies and Deaf Education, e um artigo em cada um dos periódicos: Ear and Hearing, International Journal of Pediatric Otorrinolaryngoly, Otology and Neurotology, Otolaryngology - Head and Neck Sugery e Cochlear Implants International.

Dentre os vários países participantes, pesquisadores australianos apresentaram maior número de publicações; europeus, chineses e norte-americanos representam os demais participantes. Ressalta-se que em seis publicações, os participantes foram recrutados em mais de um centro de IC; e um dos artigos foi desenvolvido com pesquisadores de três países (Alemanha, Itália e Áustria) (Quadro 1).

\section{Tamanho da amostra}

Na base de dados Medline, a amostra nas pesquisas variou entre 63 e 247 participantes dentro de uma abordagem quantitativa; e entre 13 e 27, nos estudos qualitativos. Na base de dados SciELO, a amostra foi de oito pais na abordagem quali-quantitativa e de dez pais na abordagem qualitativa (Quadro 1).

A heterogeneidade no tamanho da amostra nesses diferentes estudos indica a dificuldade de seleção de participantes com características semelhantes. A intersecção de três fatores poderia justificar esta dificuldade: quantidade de variáveis que podem interferir no desempenho da criança usuária de IC para formar um grupo homogêneo (características sócio-demográficas, etiologia, tempo de privação sensorial entre outros); o consentimento e a disponibilidade dos pais para participar dos estudos; e, restrição de uma única instituição para desenvolver a pesquisa.

\section{Tipo de pesquisa}

Observou-se que há mais estudos de abordagem quantitativa (seis), seguidos de estudos com abordagem híbrida ou quali-quantitativa (cinco) e em menor frequência, a abordagem qualitativa (dois). Mesmo sendo de natureza diferente, essas abordagens científicas se complementaram ${ }^{(13)}$. A qualitativa possibilitou o conhecimento dos valores, crenças e opiniões específicos de cada participante dos estudos analisados, muito importante para a prática clínica, especialmente na área da (re)habilitação aurioral, na qual nos deparamos com inúmeras variáveis. E, a quantitativa evidenciou as tendências observadas pelos grupos de pais, que pode auxiliar no estabelecimento das perspectivas da evolução da criança usuária de IC.

Houve predomínio de estudos transversais (11); e, dois estudos foram do tipo caso controle (Quadro 1).

As entrevistas foram predominantemente estruturadas (sete), seguidas de não estruturadas (quatro); e, em dois estudos, os dois tipos de entrevista foram realizados. O instrumento mais utilizado nas entrevistas foi o questionário (oito), seguido do formulário (três). Em dois estudos, ambos os instrumentos foram utilizados.

\section{Instrumentos}

Em diversos estudos selecionados, questionários e formulários foram utilizados nas entrevistas não estruturadas e organizados para coleta de: dados sócio-demográficos; informações que os pais têm sobre o IC, os riscos e restrições que envolvem o IC; os benefícios do IC; expectativas dos pais antes e após a criança receber o IC; modalidade comunicativa utilizada pela criança e seus pais; produção de fala e de linguagem; alterações de comportamentos; relacionamento da criança com os colegas; independência; processo de tomada de decisão; as experiências e percepções dos resultados da comunicação, pessoal, social e educacional após implante coclear ${ }^{(16,18-20,22)}$. 
Quadro 1. Informações acadêmicas e científicas dos estudos

\begin{tabular}{|c|c|c|c|c|c|c|c|c|c|c|}
\hline \multirow[t]{2}{*}{ Autores (ano) } & \multirow[t]{2}{*}{ Periódico (País) } & \multirow{2}{*}{$\begin{array}{l}\mathrm{N}^{\circ} \text { de } \\
\text { inst. }\end{array}$} & \multicolumn{2}{|l|}{$\begin{array}{c}\text { Abordagem } \\
\text { (n) }\end{array}$} & \multicolumn{2}{|c|}{$\begin{array}{l}\text { Tipo de } \\
\text { pesquisa }\end{array}$} & \multicolumn{2}{|c|}{ Entrevista } & \multicolumn{2}{|c|}{ Instrumento } \\
\hline & & & Qt & QI & Trans & $\mathrm{CC}$ & Est & NE & Qs & Form \\
\hline $\begin{array}{l}\text { Mota et al. } \\
(2011)^{(14)}\end{array}$ & Arq Int Otorrinolaringol. (Brasil) & 1 & $\begin{array}{c}\sqrt{ } \\
(\mathrm{GC}=4 ; \mathrm{GP}=4)\end{array}$ & $\sqrt{ }$ & & $\sqrt{ }$ & $\sqrt{ }$ & & $\sqrt{ }$ & \\
\hline $\begin{array}{l}\text { Ramirez-Inscoe; } \\
\text { Moore }(2011)^{(15)}\end{array}$ & Ear and Hearing. (Reino Unido) & 1 & $\begin{array}{c}\sqrt{ } \\
(\mathrm{GC}=25 ; \mathrm{GP}=23)\end{array}$ & & & $\sqrt{ }$ & $\sqrt{ }$ & & $\sqrt{ }$ & \\
\hline $\begin{array}{l}\text { Teschendorf et al. } \\
(2011)^{(16)}\end{array}$ & Otol Neurotol. (Alemanha) & 1 & $\begin{array}{c}\sqrt{ } \\
(\mathrm{N}=93) \\
\end{array}$ & & $\sqrt{ }$ & & $\sqrt{ }$ & & $\sqrt{ }$ & \\
\hline $\begin{array}{l}\text { Wang et al. } \\
(2011)^{(17)}\end{array}$ & Otol Neurotol. (República da China) & 7 & $\begin{array}{c}\sqrt{ } \\
(177)\end{array}$ & & $\sqrt{ }$ & & $\sqrt{ }$ & & $\sqrt{ }$ & \\
\hline $\begin{array}{l}\text { Hyde; Punch } \\
(2011)^{(18)}\end{array}$ & Am Ann Deaf. (Australia) & 3 & $\begin{array}{c}\sqrt{ } \\
(247)\end{array}$ & $\begin{array}{c}\sqrt{ } \\
(27)\end{array}$ & $\sqrt{ }$ & & & $\sqrt{ }$ & & $\sqrt{ }$ \\
\hline $\begin{array}{l}\text { Hyde et al. } \\
(2010)^{(19)}\end{array}$ & Am Ann Deaf. (Australia) & 3 & $\begin{array}{c}\sqrt{ } \\
(247) \\
\end{array}$ & $\begin{array}{c}\sqrt{ } \\
(27) \\
\end{array}$ & $\sqrt{ }$ & & $\sqrt{ }$ & $\sqrt{ }$ & $\sqrt{ }$ & $\sqrt{ }$ \\
\hline $\begin{array}{l}\text { Hardonk et al. } \\
(2010)^{(20)}\end{array}$ & Am Ann Deaf. (Bélgica) & 1 & & $\begin{array}{c}\sqrt{ } \\
(13)\end{array}$ & $\sqrt{ }$ & & & $\sqrt{ }$ & & $\sqrt{ }$ \\
\hline $\begin{array}{l}\text { Hyde et al. } \\
(2010)^{(21)}\end{array}$ & $\begin{array}{l}\text { J Deaf Stud. and Deaf Educ. } \\
\text { (Austrália) }\end{array}$ & 3 & $\begin{array}{c}\sqrt{ } \\
(247) \\
\end{array}$ & $\begin{array}{c}\sqrt{ } \\
(27) \\
\end{array}$ & $\sqrt{ }$ & & & $\sqrt{ }$ & $\sqrt{ }$ & $\sqrt{ }$ \\
\hline $\begin{array}{l}\text { Huttunen; } \\
\text { Välimaa (2010)(22) }\end{array}$ & $\begin{array}{l}\text { J Deaf Stud and Deaf Educ. } \\
\text { (Finlândia) }\end{array}$ & 1 & $\begin{array}{c}\sqrt{ } \\
(18) \\
\end{array}$ & $\begin{array}{c}\sqrt{ } \\
(18)\end{array}$ & $\sqrt{ }$ & & $\sqrt{ }$ & $\sqrt{ }$ & $\sqrt{ }$ & \\
\hline $\begin{array}{l}\text { May-Mederake } \\
\text { et al. }(2010)^{(23)}\end{array}$ & $\begin{array}{l}\text { Int J Pediatr Otorhinolaryngol. } \\
\text { (Alemanha; Itália; Áustria) }\end{array}$ & 7 & $\begin{array}{c}\sqrt{ } \\
(63) \\
\end{array}$ & & $\sqrt{ }$ & & $\sqrt{ }$ & & $\sqrt{ }$ & \\
\hline $\begin{array}{l}\text { Percy-Smith } \\
(2010)^{(24)}\end{array}$ & Cochlear Implants Int. (Dinamarca) & 1 & $\begin{array}{c}\sqrt{ } \\
(168)\end{array}$ & & $\sqrt{ }$ & & $\sqrt{ }$ & & $\sqrt{ }$ & \\
\hline $\begin{array}{l}\text { Loy et al. } \\
(2010)^{(25)}\end{array}$ & Otolaryngol Head Neck Sug. (USA) & 2 & $\begin{array}{c}\sqrt{ } \\
(\mathrm{GC}=918 ; \mathrm{GP}=88)\end{array}$ & & $\sqrt{ }$ & & $\sqrt{ }$ & & $\sqrt{ }$ & \\
\hline $\begin{array}{l}\text { Yamanaka et al. } \\
(2010)^{(26)}\end{array}$ & Psic Teor e Pesq. (Brasil) & 1 & & $\begin{array}{c}\sqrt{ } \\
(10)\end{array}$ & $\sqrt{ }$ & & & $\sqrt{ }$ & & $\sqrt{ }$ \\
\hline
\end{tabular}

Legenda: $\sqrt{ }=\operatorname{sim} ; \mathrm{Qt}=$ quantitativa; $\mathrm{Ql}=$ qualitativa; Trans = transversal; $\mathrm{CC}=$ caso controle; Est = estruturada; $\mathrm{NE}$ = não estruturada; $\mathrm{Qs}=$ questionário; Form = for mulário; $\mathrm{n}$ = número de pais participantes; $\mathrm{GC}$ = grupo controle; $\mathrm{GP}=$ grupo pesquisa; $\mathrm{N}^{\circ}$ de inst. = número de instituições envolvidas

Estes instrumentos possibilitaram o aprofundamento no tema proposto pelos autores, apontando os detalhes relevantes e as diversidades na amostra.

Ressalta-se que informações sócio-demográficas foram utilizadas na maioria dos estudos. São consideradas como fatores que influenciam os resultados da efetividade de programas de intervenção; mais especificamente, o parâmetro sócio-demográfico, destacando a escolaridade dos pais, é capaz de interferir de maneira relevante nos resultados, já que a acessibilidade aos sons de fala, conquistada após a ativação do IC, deve estar integrada com as possibilidades de estimulação disponíveis no ambiente no decorrer do processo de (re)habilitação aurioral ${ }^{(27)}$.

Os instrumentos utilizados para coleta de dados nas entrevistas estruturadas consistiam de escalas, questionários e formulários referenciados frequentemente nas publicações e com propriedades psicométricas analisadas (Quadro 2). Verificou-se que nos artigos internacionais, as entrevistas estruturadas foram realizadas com vários instrumentos; porém, a maioria destes ainda não foi traduzida e adaptada para o Português Brasileiro. São eles: Revised Category of Auditory Performance (CAPR); KINDL-R Questionnaire; Children's Communication Checklist (CCC-2) e LittlEARS Questionnaire (LEAQ).
Autores relacionaram os resultados do desempenho auditivo e linguístico das crianças usuárias de IC por meio das medidas diretas (coletadas pelos pesquisadores), com as informações obtidas com os pais. Em todos, evidenciou-se que há concordância entre os resultados obtidos na avaliação clínica e os obtidos com as informações dos pais, sugerindo que os pais são bons informantes ${ }^{(15,16,24)}$.

\section{Análise dos resultados dos estudos selecionados}

Os subtemas mais abordados nas pesquisas foram "desempenho auditivo e/ou de linguagem de crianças usuárias de IC" (seis artigos) e "perspectivas e/ou expectativas dos pais sobre o IC antes da cirurgia" (quatro artigos). Com apenas um artigo, os demais subtemas foram: "aspectos educacionais", "efetividade do programa" e "qualidade de vida".

\section{Perspectivas elou expectativas dos pais sobre o IC antes da cirurgia}

Sabe-se que o processo de decidir ou não pelo IC é difícil para os pais. A maioria deles demorou menos de três meses para tomada de decisão; outros levaram entre três e seis meses. 
Quadro 2. Instrumentos utilizados nas entrevistas estruturadas

\begin{tabular}{|c|c|c|}
\hline Autores & Instrumento & Medida \\
\hline Hyde et al. ${ }^{(19,21)}$ & $\begin{array}{l}\text { Questionário com } 33 \text { itens, divididos } \\
\text { em cinco subescalas para medir as } \\
\text { expectativas das mães }\end{array}$ & $\begin{array}{l}\text { Habilidades de Comunicação, Habilidades Sociais, Conquistas Acadêmicas, } \\
\text { Mudança na Vida Futura e Demandas para Reabilitação. Para os itens em cada } \\
\text { subscale da secção do questionário foi solicitado para os pais avaliar o nível de } \\
\text { concordância sobre as suas expectativas antes de seu filho receber o implante } \\
\text { coclear. }\end{array}$ \\
\hline $\begin{array}{l}\text { Percy-Smith }{ }^{(24)} \text {; } \\
\text { Wang et al. }{ }^{(17)}\end{array}$ & $\begin{array}{l}\text { Revised Category of Auditory } \\
\text { Performance (CAPR) }\end{array}$ & $\begin{array}{l}\text { Medida padronizada, na qual o nível mais baixo descreve nenhuma consciência } \\
\text { aos sons ambientais - zero - e o nível mais alto - nove - é representado pela } \\
\text { capacidade de falar com estranhos no telefone }\end{array}$ \\
\hline Mota et al. ${ }^{(14)}$ & $\begin{array}{l}\text { Lista de Avaliação do Vocabulário } \\
\text { Expressivo (LAVE) }\end{array}$ & $\begin{array}{l}\text { Registro do número de vocábulos de diferentes categorias produzidos } \\
\text { espontaneamente pela criança }\end{array}$ \\
\hline Loy et al. ${ }^{(25)}$ & $\begin{array}{l}\text { KINDL-R Questionnaire para medir } \\
\text { a qualidade de vida, versão para } \\
\text { criança (entre } 8 \text { e } 11 \text { anos de idade) } \\
\text { e para pais }\end{array}$ & $\begin{array}{l}24 \text { questões igualmente distribuídas em } 6 \text { subescalas: bem estar físico, bem estar } \\
\text { psicológico, auto-estima, família, amigos e escola }\end{array}$ \\
\hline $\begin{array}{l}\text { Ramirez-Inscoe; } \\
\text { Moore }^{(15)}\end{array}$ & $\begin{array}{l}\text { Children's Communication Checklist } \\
\text { (CCC-2) }\end{array}$ & $\begin{array}{l}\text { Instrumento de triagem que identifica criança com risco para comprometimento } \\
\text { de linguagem }\end{array}$ \\
\hline $\begin{array}{l}\text { Teschendorf } \\
\text { et al. }{ }^{(16)}\end{array}$ & $\begin{array}{l}\text { Student Oral Language Observation } \\
\text { Matrix (SOLOM) }\end{array}$ & Questionário que avalia a competência da criança em sua língua nativa \\
\hline Percy-Smith ${ }^{(24)}$ & Speech Intelligibility Rating (SIR) & Medida da inteligibilidade de fala na conversação entre diferentes sujeitos. \\
\hline $\begin{array}{l}\text { May-Mederake } \\
\text { et al. }{ }^{(21)}\end{array}$ & Questionário LittIEARS (LEAQ) & $\begin{array}{l}\text { Testa habilidades linguísticas receptiva, semantica e expressiva em resposta à } \\
\text { entrada auditiva }\end{array}$ \\
\hline
\end{tabular}

As expectativas, esperanças e crenças dos pais sobre o IC podem refletir mais precisamente a relação pais-filho, na busca determinada em fazer o melhor para seus filhos ${ }^{(21)}$.

Os aspectos que interferiram na decisão dos pais e que estavam em vários momentos associados com a urgência em ter a sua criança implantada podem ser classificados como: quais os encaminhamentos e orientações os pais receberam dos profissionais; aspectos biomédicos (riscos na cirurgia; dispositivo; cuidados pós-cirurgicos; (re)habilitação e éticos (opinião sobre definição de desvantagem; cirugia em crianças muito pequenas; risco de vida; opinião da criança; identidade surda); qualidade, quantidade e diversidade das informações sobre o programa completo; conhecimentos sobre os cuidados com o dispositivo; o processo de reabilitação; experiência anterior com pessoas surdas; apoio social; e, custos envolvidos no processo ${ }^{(20)}$.

Em uma pesquisa nacional, o IC foi descrito pelos pais como uma solução não apenas para a surdez de seu filho, mas como uma possibilidade de ele ter um futuro melhor na escola, no trabalho e nos relacionamentos; torná-lo independente e também uma esperança de melhoria na qualidade de vida da criança. A maioria dos pais já conhecia de alguma maneira o IC antes de chegar ao ambulatório do centro de IC; informações estas obtidas de diversas fontes. Os pais estavam cientes que o IC poderia ocasionar dificuldades tanto para a criança (na adaptação ao uso da unidade externa), quanto para a família (presenciar uma nova situação que gera angústia e desespero). Por outro lado, acreditavam que haveria benefícios com a melhoria da comunicação e a possibilidade do filho ouvir e falar ${ }^{(26)}$.

Inseridos no programa de IC ou na (re)habilitação aurioral, os pais podem utilizar uma série de fontes de informação para esclarecer suas dúvidas para a tomada de decisão sobre seu filho receber ou não o IC. Pesquisadores australianos identificaram que uma das principais fontes de informação foi a própria equipe de profissionais do centro de IC: os audiologistas $(40,9 \%) \mathrm{e}$ os otorrinolaringologistas $(23,1 \%)$. Outras 13 possíveis fontes de informação também foram citadas pelos pais. Destas, destacaram-se: outras famílias com crianças implantadas, as próprias crianças usuárias de IC e professores. Poucos contataram a Comunidade Surda ou Organizações de Pessoas Surdas. Os pais relataram ciência da opinião negativa dos membros desta comunidade sobre o IC. A tomada de decisão foi estressante para os pais; contudo, na medida em que se tornava mais fácil, eles acreditavam que o IC poderia ser "a única opção" para a criança desenvolver a audição e fala e se comunicar na mesma modalidade com os outros membros da família ${ }^{(21)}$.

Há um elevado grau de coerência entre as expectativas dos pais e as experiências posteriores à cirurgia. Em um estudo, os resultados quantitativos indicaram que a maioria dos pais pensava no IC como um passo positivo na vida de seus filhos antes da cirurgia; e, posteriormente, altos níveis de satisfação nos resultados finais - pós cirurgia foram alcançados. Os autores consideraram também, na análise qualitativa, as diversidades nas respostas: alguns pais não demonstraram satisfação com os resultados das habilidades comunicativas, sociais e acadêmicas de seus filhos; eram pais cujos filhos tinham outros comprometimentos associados, que os impediam de se beneficiar com seus implantes ${ }^{(19)}$.

\section{Desempenho auditivo elou de linguagem de crianças usuárias de IC}

Os pais observaram que as crianças usuárias de IC se beneficiaram com o acesso aos sons da fala e, consequentemente, 
a probabilidade de desenvolver a língua falada em menor intervalo de tempo foi maior quando comparado com o uso de prótese auditiva(23).

Os relatos dos pais sobre o desempenho auditivo em crianças usuárias de IC fornecem uma medida de funcionamento na vida real delas. E, para responder quais são os fatores preditivos para atingir desempenhos auditivos satisfatórios, pesquisadores chineses desenvolveram um estudo e identificaram os seguintes fatores: horas de uso do dispositivo por dia (16-24 horas); a modalidade comunicativa (oral/aural) em casa e na escola; abordagem educacional (sala regular); e, sem comprometimentos associados. Concluíram que a percepção dos pais em todo o processo deve ser examinada, tanto na tomada de decisão sobre o IC para seus filhos, como no seguimento após cirurgia ${ }^{(17)}$.

Num estudo dinamarquês ${ }^{(24)}$, os pais pontuaram em uma escala o nível das habilidades auditivas, linguísticas e comunicativas que seus filhos se encontravam. Os resultados indicaram que $81 \%$ das crianças usuárias de IC eram no mínimo capazes de entender frases comuns sem leitura facial nas situações do dia-a-dia, e em alguns casos, capazes de compreender um falante conhecido no telefone, após três anos de uso do IC. As informações dos pais sobre a inteligibilidade da fala dos filhos indicaram que a maioria das crianças (69\%) era compreendida por qualquer um nas situações do dia-a-dia.

As observações não são necessariamente pontuais. Os pais podem relatar em diferentes momentos (antes e após a ativação do IC) as mudanças comportamentais observadas em seus filhos. Em um estudo, os pais responderam o questionário seis meses e depois anualmente (um a cinco anos) após a ativação. Os resultados sugeriram que o desenvolvimento das habilidades auditivas, após o acesso aos sons da fala promovido pelo IC, possibilitou em $77 \%$ das crianças melhora significativa no uso da fala entre o primeiro e segundo ano após ativação, mudança na modalidade comunicativa, bem como maior facilidade para a criança se comunicar com os pais e os irmãos. Os autores verificaram também que houve diminuição do uso de sinais e da dependência do contato de olho; e, observaram vários benefícios sócio emocionais (autoconfiança, segurança, expansão na vida social) e comportamentais (criança mais calma). Após cinco anos da ativação, a maioria dos pais demonstrou satisfação com o desenvolvimento auditivo e de linguagem de seus filhos ${ }^{(22)}$.

Competências lingüísticas avançadas, incluindo a aprendizagem de uma segunda língua falada, são possíveis para as crianças com IC vivendo em lares bilíngues. Porém, os resultados de um estudo desenvolvido na Alemanha ${ }^{(16)}$ sugeriram que tal desempenho é exceção e não regra para a população infantil usuária de IC, pois crianças expostas a uma língua (alemão) demonstraram resultados melhores no teste do que as crianças do grupo bilingue nos cinco momentos testados (até 36 meses) após ativação do IC.

Com os programas de detecção da perda auditiva no período neonatal e o início da intervenção mais precoce em bebes, houve necessidade de utilizar novos instrumentos para monitorar o desenvolvimento das habilidades auditivas das crianças pequenas, inclusive aquelas com outros comprometimentos associados e usuárias de IC. Pesquisadores europeus ${ }^{(23)}$, por meio da bateria de teste LittlEARS (LEAQ), realizaram um estudo longitudinal com crianças usuárias de IC até o segundo ano de vida e compararam os resultados com o grupo de crianças ouvintes. Os resultados demonstraram que as crianças usuárias de IC com idade inferior a 12 meses foram significativamente melhores do que o grupo etário mais velho (entre 12 e 36 meses). Todas as crianças com IC atingiram a pontuação máxima possível no LEAQ, em média, com 22 meses de idade auditiva. Três crianças com necessidades especiais mostraram um crescimento mais lento ao longo do tempo, mas também foram beneficiadas com a indicação precoce do IC.

Na prática clínica pode-se observar uma grande variabilidade de resultados, uma vez que nem todas as crianças apresentam resultados satisfatórios, como descritos anteriormente. Neste levantamento bibliográfico, um dos estudos chamou atenção para esta questão. Foi realizada a comparação do desempenho de dois grupos de crianças usuárias de IC, sendo um com desenvolvimento de linguagem típico e outro com desenvolvimento de linguagem desproporcional (DLI); com mais dois grupos de crianças ouvintes, ou seja, os irmãos das crianças dos grupos anteriores. Os resultados indicaram que as dificuldades para o desenvolvimento da linguagem não são determinadas apenas pela surdez e/ou pelas características do dispositivo, mas sim por meio dos mesmos fatores hereditários e ambientais que influenciam o desenvolvimento da linguagem nas crianças ouvintes, visto que a maioria dos irmãos ouvintes do grupo DLI também apresentaram desenvolvimento de linguagem atípico ${ }^{(15)}$.

\section{Outros subtemas}

Um fator preditivo importante que deve ser considerado para o sucesso nos resultados é a qualidade do serviço prestado no programa de (re)habilitação e o tempo (número de sessões) necessário para atingir a meta, ou seja, a efetividade de um programa. Tal conclusão ficou evidenciada num estudo nacional $^{(14)}$, com dois grupos de pais: um, que frequentou sessões de orientação e outro, que não frequentou. A análise quantitativa evidenciou que quatro sessões de orientação aos pais, abordando temas sobre as habilidades auditivas e linguísticas, não foram suficientes para observar mudança significativa na quantidade de vocábulos produzidos espontaneamente pelas crianças; porém, qualitativamente, a média de palavras adquiridas pelas crianças no grupo de pais participantes foi maior que no grupo não participante.

Os estudos não estão focados apenas nos resultados clínicos, mas também na vida social da criança usuária de IC. Novas pesquisas surgem com foco nos comportamentos e adaptações psicossociais, ou seja, estudos da qualidade de vida das crianças e seus familiares. Pesquisadores americanos ${ }^{(25)}$ examinaram os resultados do questionário de qualidade de vida relativo à saúde de crianças usuárias de implante coclear e compararam com as de crianças ouvintes, pareadas por idade, e com seus pais. Verificaram que o grupo de crianças usuárias de IC classificou sua qualidade de vida $(\mathrm{QV})$ de forma menos positiva (média da pontuação igual a 72,6), quando comparada com o grupo de crianças ouvintes (média da pontuação igual a 83,98). As crianças com IC tendem a relatar QV mais positiva nos domínios de bem estar emocional e autoestima. As comparações entre as crianças 
usuárias de IC e seus pais indicaram resultados similares em todas as subescalas e no resultado total. Portanto, provou-se que os pais são relatores confiáveis nas áreas em que eles podem observar o comportamento de seus filhos, que o IC parece ter efeito positivo em certos domínios psicológicos e parece não criar graves problemas globais psicológicos em seus usuários.

Geralmente a primeira meta dos pais e dos profissionais da reabilitação da criança com IC é possibilitar o acesso aos estímulos de fala suficiente para a criança desenvolver as habilidades auditivas e, consequentemente, a língua oral. Contudo, há a possibilidade de utilização de uma abordagem bilíngue e que os centros disponibilizem para os pais serviços nos quais eles possam escolher para seus filhos modalidades comunicativas diferentes, isto é, ofereceram a modalidade aurioral e a língua de sinais para as crianças. Para entender como as crianças usuárias de IC lidam com estas duas modalidades comunicativas, pesquisadores australianos ${ }^{(18)}$ examinaram um grupo de crianças usuárias de fala e língua de sinais, de diferentes faixas etárias, na perspectiva dos pais. Verificaram que $87 \%$ deles escolheram o IC para os seus filhos como um meio para desenvolver a comunicação por meio da audição e fala; $12 \%$ indicaram que queriam que seus filhos usassem uma combinação de fala e sinais. Dos 27 pais entrevistados, 19 estavam usando sinais em algum grau com seus filhos. Estes justificaram que os benefícios implicariam na ajuda para as crianças desenvolverem a linguagem, proporcionando um meio de comunicação quando elas não estavam usando seu IC, e possibilitando a participação de seus filhos nos meios sociais e na construção de sua identidade cultural surda. Relativo às crianças, os resultados indicaram que elas alternaram prontamente entre os modos de comunicação conforme o parceiro: falante ou sinalizador. Alguns pais relataram que o uso de sinais antes da cirurgia contribuiu para o desenvolvimento da fala de seus filhos. Por outro lado, houve relatos sobre a dificuldade dos pais aprender a língua de sinais. A fala e os sinais podem fazer parte do dia a dia de muitas crianças, com o potencial de beneficiar o desenvolvimento da língua falada, o desenvolvimento social e participação nas suas comunidades.

Os resultados dos estudos selecionados evidenciaram a importância da participação dos pais para monitorar a evolução das crianças usuárias de IC num contexto natural (ecológico) tanto na tomada de decisão quanto à candidatura da criança, como no processo de (re)habilitação aurioral, nas diferentes faixas etárias da criança. Nestes contextos, os fatores, que podem intervir no processo terapêutico fonoaudiológico, devem ser considerados (Quadro 3).

A análise dos estudos sobre os diferentes domínios da participação dos pais nos programas de (re)habilitação aurioral de crianças usuárias de IC torna-se necessária para que o fonoaudiólogo compreenda como ocorre o engajamento dos pais, bem como para ter acesso aos instrumentos clínicos para identificar as suas expectativas e analisar suas observações. Tais ações possibilitarão construir um plano terapêutico e aprimorar a qualidade do serviço fonoaudiológico prestado aos pais.

A integração das informações do conjunto dos 13 estudos possibilitou a identificação dos principais subtemas: "desempenho auditivo e/ou de linguagem de crianças usuárias de IC"
Quadro 3. Síntese dos fatores que interferem na participação dos pais do programa de implante coclear

\begin{tabular}{|c|c|}
\hline Condições & Fatores \\
\hline $\begin{array}{l}\text { Tomada de } \\
\text { decisão }\end{array}$ & $\begin{array}{l}\text { - O que os pais já sabem sobre IC } \\
\text { - Expectativas } \\
\text { - Informações recebidas: qualidade e quantidade } \\
\text { - Encaminhamentos realizados } \\
\text { - Aspectos biomédicos e éticos } \\
\text { - Inserção na (re)habilitação } \\
\text { - Contato com: outros pais, usuários de IC, } \\
\text { pessoas surdas, comunidade surda } \\
\text { - Apoio social } \\
\text { - Custos }\end{array}$ \\
\hline $\begin{array}{l}\text { Fatores } \\
\text { preditivos }\end{array}$ & $\begin{array}{l}\text { - Tempo de uso diário do IC } \\
\text { - Modalidade Comunicativa } \\
\text { - Tipo de escola } \\
\text { - Comprometimentos associados } \\
\text { - Aspectos sócio-demográficos } \\
\text { - Efetividade do programa de intervenção }\end{array}$ \\
\hline $\begin{array}{l}\text { Benefícios e } \\
\text { limitações }\end{array}$ & $\begin{array}{l}\text { - Modalidade comunicativa: aurioral e/ou viso- } \\
\text { espacial } \\
\text { - Evolução das habilidades auditivas e linguísticas } \\
\text { - Possibilidade de uma segunda língua oral } \\
\text { - Benefício sócio-emocional e comportamental }\end{array}$ \\
\hline Satisfação & - Qualidade de vida \\
\hline
\end{tabular}

e "perspectivas e/ou expectativas dos pais sobre o IC antes da cirurgia". A interpretação dos resultados, sejam eles positivos ou negativos, levou em consideração: as condições dos pais na tomada de decisão; os diferentes fatores preditivos do desenvolvimento; os benefícios e limitações com o acesso aos sons da fala com o IC; e, o grau de satisfação dos pais.

Esta revisão de literatura apresentou algumas limitações: a restrição à língua portuguesa brasileira e a inglesa, pois na primeira fase da seleção do material, vários artigos foram citados na língua não inglesa. $\mathrm{E}$, o período selecionado para a coleta - 18 meses - provavelmente gerou um menor alcance dos trabalhos desenvolvidos com esta temática. A revisão sistemática proposta neste estudo não teve a intenção de focar e aprofundar em um aspecto da participação dos pais no programa de IC; mas, identificar e compreender a amplitude da influência dos pais nas diferentes etapas da (re)habilitação.

Novas investigações são necessárias, para a adaptação e validação para o Português Brasileiro de questionários ou formulários reconhecidos internacionalmente, bem como a busca de evidências dos fatores identificados. Recomenda-se também que pesquisas multicêntricas com maior amostragem sejam desenvolvidas, bem como a continuidade de pesquisas com rigoroso nível de evidência científica.

\section{CONCLUSÃO}

A revisão sistemática proposta neste estudo indicou que o tema participação dos pais de crianças usuárias de IC é pesquisado por estudiosos do mundo todo, entre eles australianos, europeus, americanos, chineses e brasileiros, e que 
seus respectivos estudos estão disponíveis na base de dados SciELO e Medline.

Observou-se um predomínio de estudos transversais e caso controle por meio de entrevistas qualitativas e/ou quantitativas. Destaca-se que aqueles que tinham mais participantes, eram publicações cuja amostra foi coletada em mais de um centro de programa de IC.

Foram identificados os fatores sobre a tomada de decisão em realizar ou não a cirurgia (conhecimento prévio sobre IC, expectativas dos resultados, qualidade e quantidade de informações, encaminhamentos realizados, aspectos biomédicos e éticos, inserção na reabilitação, contato com outros pais, apoio social e custos); os fatores preditivos relacionados aos resultados da reabilitação (tempo de uso diário do IC, modalidade aurioral, escola regular, comprometimentos associados, aspectos sócio-demográficos e efetividade do programa); os fatores relacionados aos benefícios e limitações do IC (modalidade comunicativa, evolução das habilidades auditivas e linguísticas, o aprendizado de uma segunda língua oral), bem como o grau de satisfação dos pais, com os resultados relacionados à qualidade de vida.

* MIVC foi responsável pela concepção e delineamento do tema; coleta e análise dos dados; redação do artigo; aprovação da versão final. ACMC contribuiu com a metodologia do estudo, análise, revisão crítica sobre o conteúdo e conclusão dos achados; redação do artigo; aprovação da versão final.

\section{REFERÊNCIAS}

1. Bevilacqua MC, Formigoni GMP. Audiologia educacional: uma opção terapêutica para a criança deficiente auditiva. Carapicuíba: Pro-Fono; 1997.

2. Tye-Murray N. Foundations of aural rehabilitation: children, adults and their family members. 2nd ed. New York: Thomson Learning Inc.; 2004.

3. Madell JR, Flexer C. Pediatric audiology: diagnosis, technology, and management. New York: Thieme; 2008. Communication approaches for managing hearing loss in infants and children. p. 205-9.

4. Fernandes FDM, Mendes BCA, Navas ALGP. Tratado de fonoaudiologia. 2a ed. São Paulo: Roca; 2009. Implante Coclear. p. 220-31.

5. Moeller MP. Early intervention and language development in children who are deaf and hard of hearing. Pediatrics. 2000 Sep;106(3):E43.

6. Yoshinaga-Itano C. Levels of evidence: universal newborn hearing screening (UNHS) and early hearing detection and intervention systems (EHDI). J Commun Disord. 2004 Sep;37(5):451-65.

7. Fitzpatrick E, Angus D, Durieux-Smith A, Graham ID, Coy D. Parents' needs following identification of childhood hearing loss. Am J Audiol. 2008 Jun;17(1):38-49.

8. Sass-Lehrer M. Early beginnings for families with deaf and hard of hearing children: myths and facts of early intervention and guidelines for effective services. Washington, DC: Laurent Clerc National Deaf Education Center - Gallaudet University; 2002.
9. Cox RM. Waiting for evidence-based practice for your hearing aid fittings? It's here! Hear J. 2004;57(8):10,12,14,16,17.

10. Mendes TM, Alvarenga KF. Capacitação de profissionais da saúde na área de saúde auditiva: revisão sistemática. Rev Soc Bras Fonoaudiol. 2009;14(2):280-6.

11. Tanamati LF, Costa OA, Bevilacqua MC. Resultados a longo prazo com o uso de implante coclear em crianças: revisão sistemática. Arq Int Otorrinolaringol. 2011;15(3):365-75.

12. Marconi MA, Lakatos EM. Fundamentos de metodologia científica. $6 a$ ed. São Paulo: Atlas; 2007.

13. Minayo MCS, Sanches, O. Quantitativo - qualitativo: oposição ou complementaridade? Cad. Saúde Pública. 1993 Jul-Set;9(3):239-48.

14. Mota JM, Tavares TF, Koji RT, Bento RF, Matas CG, Andrade CRF, et al. Efeito do programa de orientação a pais no desenvolvimento lexical de crianças usuárias de implante coclear. Arq Int Otorrinolaringol. 2011 Mar;15(1):54-8.

15. Ramirez-Inscoe J, Moore DR. Processes that influence communicative impairments in deaf children using cochlear implants. Ear Hear. 2011 Nov-Dec;32(6):690-8.

16. Teschendorf M, Janeschik S, Bagus H, Lang S, Arweiler-Harbeck D. Speech development after cochlear implantation in children from bilingual homes. Otol Neurotol. $2011 \mathrm{Feb} ; 32(2): 229-35$.

17. Wang NM, Liu CJ, Liu SY, Huang K, Kuo YC. Predictor of auditory performance in mandarin chinese children with cochlear implants. Otol Neurotol. 2011;32(6):937-42.

18. Hyde M, Punch R. The modes of communication used by children with cochlear implants and the role of sign in their lives. Am Ann Deaf. 2011;155(5):535-49.

19. Hyde M, Punch R, Komesaroff L. A comparison of the anticipated benefits and received outcomes of pediatric cochlear implantation: parental perspectives. Am Ann Deaf. 2010;155(3):322-38.

20. Hardonk S, Bosteels S, Desnerck G, Loots G, Hove GV, Kerschaver EV, Van Roelen CV, Louckx A. Pediatric cochlear implantation: a qualitative study of parental decision making process. Am Ann Deaf. 2010;155(3):339-52.

21. Hyde M, Punch R, Komesaroff L. Coming to a decision about cochlear implantation: parents making choices for their deaf children. J Deaf Stud Deaf Educ. 2010;15(2):162-78.

22. Huttunen K, Välimaa T. Parents' views on changes in their child's communication and linguistic and socio emotional development after cochlear implantation. J Deaf Stud Deaf Educ. 2010;15(4):383-404.

23. May-Mederake B, Kuehn H, Vogel A, Keilmann A, Bohnert A, Mueller $\mathrm{S}$, et al. Evaluation of auditory development in infants and toddlers who received cochlear implants under the age of 24 months with the LittlEARS ( Auditory Questionnaire. Int J Pediatr Otorhinolaryngol. 2010;74(10):1149-55.

24. Percy-Smith L. Associations between auditory capacity, speech and language, level of communication and parental assessment of children with cochlear implant. Cochlear Implants Int. 2010;11(1):50-62.

25. Loy B, Warner-Czyz AD, Tong L, Tobey EA, Roland PS. The children speak: an examination of the quality of life $\mathrm{f}$ pediatric cochlear implant users. Otolaryngol Head Neck Surg. 2010;142(2):247-53.

26. Yamanaka DA, Silva RB, Zanolli ML, Silva AB. Implante coclear em crianças: a visão dos pais. Psic Teoria Pesq. 2010;26(3):465-73.

27. Yehudai N, Tzach N, Shpak T, Most T, Luntz M. Demographic factors influencing educational placement of the hearing-impaired child with a cochlear implant. Otol Neurotol. 2011;32(6):943-7. 